\title{
APPLICATION OF WIRELESS COMMUNICATION TECHNOLOGY IN ELECTROMAGNETIC WAVE TRANSMISSION AND SCATTERING MACHINERY
}

\author{
Guangliang Wang \\ School of Electronic and Electrical Engineering, Zibo Vocational Institute, Zibo, 255000, China \\ Email: wglguangliang@yeah.net
}

\begin{abstract}
The study aims to apply a wireless sensor network in the field of electromagnetic wave transmission and mechanical fault prediction. A wireless short-range communication system for underground electromagnetic wave transmission is proposed based on the principle of electromagnetic induction and underground short-range wireless communication. And in the light of the principles of scattering transform and a wireless sensor network, the mechanical fault is predicted. A wireless sensor network detects and monitors mechanical faults and extracts fault features, while it predicts mechanical faults based on the signal processing of scattering transform. The relationship between EMF (Electromotive Force) of receiving coil and carrier frequency under different dielectric conductivity is analysed by MATLAB (Matrix \& Laboratory). The results show that the induced voltage first increases and then decreases with the increase of frequency under different conductivity conditions, and the optimal carrier frequency decreases with the increase of conductivity. Under the non-noise condition, the accuracy of the scattering change method used is more than 99\%, which is higher than that of the other three feature extraction methods. On different training sets, the classification performance of LSTPSVM (least squares projection double support vector machine) is higher than that of SVM (support vector machine) and PSVM (probabilistic support vector machine). The proposed wireless communication system has the ability to choose the best carrier frequency. With the increase of carrier frequency, the BER (symbol error rate) first increases and then decreases, and can effectively suppress other frequency components in the signal, extract the characteristics of periodic fault signal, so as to achieve the purpose of feature extraction. The results show that the wireless sensor network and the scattering transformation results can effectively predict the mechanical failure, and the wireless short-range communication technology can be effectively used in underground electromagnetic wave transmission.
\end{abstract}

Keywords: A wireless sensor network; Wireless short range communication system; Electromagnetic wave; Scattering transformation; Mechanical failure.

\section{Introduction}

Oil is an important energy source. Commodities and many industrial products commonly used in people's lives are extracted from oil, including the raw materials used in factories, and the fuels used in automobiles and cosmetics [1]. At present, the world's oil and natural gas resources begin to decrease sharply, and human beings gradually increase the frequency of oil exploitation. Thus, the oil and gas resources are decreasing at a faster speed, and the difficulty of the development and utilization of oil and gas resources is gradually increasing. All countries in the world are competing to innovate and improve oil exploitation technology. As resources are becoming less and less, countries also begin to reserve oil and gas resources for future use [2,3], which greatly promotes the development of the oil industry. Meanwhile, the required skills for exploiting oil and natural gas become more complicated and are difficult to acquire. And the demand for oil is continuously increasing, and oil exploitation technology and drilling technology have a huge impact on oil industry, which gradually becomes a key factor for the development of oil industry $[4,5]$.

Drilling technology is more complex than other technologies, accounting for nearly half of the investment in the oil industry, which is considered as one of the important technologies affecting the development of the oil industry. Its level and technology have a great influence on the upstream of oil drilling [6]. Due to the gradual acceleration of industrial development, the intensity and difficulty 
of drilling are gradually increasing. Various types of wells are drilled rapidly. Therefore, the drilling speed and technology are greatly advanced and improved [7]. With the rapid development of MWD (measurement while drilling) system and downhole automatic vertical drilling technology, the downhole drilling technology in the world begins to develop rapidly, and the downhole remote control variable stabilizer is successfully realized.

Furthermore, the intellectualization is introduced and the wireless short distance transmission technology of downhole near bit is the key technology of intellectualization $[8,9]$. In the rotary steering drilling system, downhole information, such as tool face angle and time collected by near bit sensor, should be transmitted to the surface in real time to facilitate surface analysis and monitoring. After the completion of surface analysis and monitoring, the mud pulse is transmitted to the surface by downward communication to realize the large closed-loop system of rotary steering drilling, which is transmitted underground to control the operation of offset actuator [10].

Wireless communication technology can also be applied to industrial monitoring system, which can analyse a series of problems caused by unpredictable factors, so as to reduce the cost of products and improve work efficiency [11]. In terms of mechanical fault diagnosis, Intel is installed 200 sensor nodes on a small number of measurable components in 4000 kinds of chip manufacturing components, which are used to monitor the vibration of equipment, and provide monitoring reports when the measurement results exceed the requirements. The effect is very significant. At the beginning of this century, China Oil Exploration and Development Research Institute designs a new type of underground short distance transmission wireless communication system.
The system takes the short distance communication machine as the core, which can be effectively used in downhole signal transmission. The data collected by the proximity sensor can be transmitted to the MWD system through wireless transmission, providing an effective communication method for downhole drilling [12].

Moreover, a wireless short distance communication system is proposed and applied to underground electromagnetic wave transmission. Meanwhile, it innovatively combines a wireless sensor network with scattering transformation, which is applied to mechanical fault prediction. The study provides experimental basis for the practical application of wireless communication technology in the field of electromagnetic wave transmission and scattering machinery.

\section{Electromagnetic Wave Transmission Design and Mechanical Fault Prediction based on Wireless Communication Technology}

\subsection{Underground Electromagnetic Wave Transmission Design based on Wireless Short Range Communication System}

First of all, electromagnetic induction and underground short-range wireless communication principle are introduced. Electromagnetic induction phenomenon [13] refers to the conductor placed in the variable magnetic flux, which produces electromotive force. This electromotive force is called induced electromotive force. If the conductor is closed into a loop, the electromotive force will drive electrons to flow and form an induced current. The relevant contents of electromagnetic induction are shown in Table 1 below:

Table 1 Contents related to electromagnetic induction

\begin{tabular}{|ll|}
\hline Knowledge types involved in electromagnetic induction & Contents \\
\hline Phenomena and rules & Faraday's law of electromagnetic induction and Lenz's law [14] \\
Roles of the right hand & $\begin{array}{l}\text { Electric energy is transmitted and distributed in the circuit, and } \\
\text { converted into other forms of energy through electrical } \\
\text { equipment. In practical application, Ohm's law, resistance law, } \\
\text { Joule's law, Newton's law, kinetic energy law and energy } \\
\text { conservation law are often used. } \\
\text { Place the right hand flat so that the thumb is perpendicular to the } \\
\text { other four fingers and in the same plane as the palm. Put the } \\
\text { right hand in the magnetic field. If the magnetic line of force } \\
\text { enters the palm vertically, the thumb is moving in the direction } \\
\text { of the wire, and the direction of the four fingers is the direction of } \\
\text { the induced current. }\end{array}$
\end{tabular}

As for the underground wireless short distance communication system, the transmission environment of electromagnetic wave is completely different from that on the ground. The underground environment is complex, and electromagnetic wave propagation in electrolyte encounters great 
obstacles. According to the electromagnetic field theory [15], the electromagnetic wave with higher frequency has great attenuation when it propagates in the electrolyte. Therefore, the selection of electromagnetic wave communication frequency is subjected greatly to the influence of the ground.

Ground communication needs the electromagnetic wave frequency with higher speed. If the underground communication is adopted, the underground transmission medium is mainly mud, which is salty. In addition, its conductivity is relatively large, and the high-frequency electromagnetic wave in the electrolyte attenuates depresses rapidly. Therefore, the transmission cannot meet the requirements, and the electromagnetic wave with lower frequency can be selected. In the light of the characteristics of signal transmission, there are analogue and digital communication modes. Compared with analogue communication, digital communication has stronger anti-jamming ability. Therefore, the digital communication is selected here [16]. In order to make the digital signal be transmitted through wireless mode, it is necessary to replace the digital signal with analogue wave, so as to facilitate the transmission in the channel and make the underground transmission more efficient.

Moreover, the electromagnetic theory of underground wireless short-range communication is analysed. When the electromagnetic theory of underground wireless short-range communication is analysed, Maxwell equations [17] should be used.

According to Maxwell complex equation of plane electromagnetic wave in infinite uniform semiconductor medium, vector wave equation can be obtained, with the propagation law being neglected. This can be expressed as the form of plane wave:

$$
\begin{aligned}
& E=E_{0} e-j(\omega-j \mu) z=E_{0} e^{-\omega q} e^{-j \mu q} \\
& \mu=\varphi \sqrt{\frac{\lambda \varepsilon}{2}\left[\sqrt{1+\left[\frac{\sigma}{\varphi \varepsilon}\right]^{2}}-1\right]} \\
& \omega=\varphi \sqrt{\frac{\lambda \varepsilon}{2}\left[\sqrt{1+\left[\frac{\sigma}{\varphi \varepsilon}\right]^{2}+1}\right]}
\end{aligned}
$$

$e^{-\omega q}$ represents the amplitude factor of electric field intensity, $\mu$ is the attenuation constant, $\omega$ is the phase constant, $\varepsilon$ is the dielectric constant, $\lambda$ is the permeability, $\sigma$ is the conductivity, and $\varphi$ is the angular frequency.
The classification of general media is determined by $\frac{\sigma}{\varphi \varepsilon}$, and the classification is shown in Table 2 below:

Table 2 Media classification

\begin{tabular}{lc}
\hline Media classification & Conditions \\
\hline Dielectric & $\frac{\sigma}{\varphi \varepsilon}<1$ \\
Bad conductor & $\frac{\sigma}{\varphi \varepsilon}=1$ \\
Good conductor & $\frac{\sigma}{\varphi \varepsilon}>1$ \\
\hline
\end{tabular}

The properties of the medium are not only related to the parameters of the medium itself, but also affected by the frequency of electromagnetic waves. According to the actual condition, when the electromagnetic wave is transmitted in the good conductor of the underground short-range communication, the attenuation constant increases with the increase of the electromagnetic wave frequency, improving the attenuation of the electromagnetic wave. Thus, if the low-frequency communication is adopted, the electromagnetic wave used in underground communication should be low-frequency electromagnetic wave. In the underground short-range communication, the induced electromotive force received by the receiving coil is not only the induced electromotive force produced by the electromagnetic wave electric field, but also the induced electromotive force produced by the magnetic field. The induced electromotive force is closely related to the distance between the two coils, the conductivity of the medium, the frequency of the electromagnetic wave and other parameters [18]. Hence, it is necessary to further analyse the induced electromotive force of the receiving coil. The induced electromotive force depends on the distance between the two coils, the conductivity of the medium and the frequency of the electromagnetic wave. Suppose the cross-sectional area of the transmitting coil is $\mathrm{A}$, the number of turns of the coil is $\mathrm{M}$, the current is Iejpt, the number of turns of the receiving coil is $\mathrm{M}^{\prime}$ ', and the crosssectional area is $S^{\prime}$, then the induced electromotive force of the receiving coil is:

$$
\begin{aligned}
& E=E^{\prime} \mathrm{e}^{-j v L}(1+\mathrm{j} v L) \\
& E^{\prime}=-\frac{I \varphi \lambda S^{\prime} M^{\prime} S M}{2 l^{3}} \\
& v=\sqrt{-j \varphi \lambda \sigma}
\end{aligned}
$$


I represents the current of the transmitting coil and $\mathrm{L}$ represents the distance between the transmitting coil and the receiving coil.

When the other parameters except $\varphi$ are constant, the induced electromotive force of the receiving coil has a nonlinear relationship with the frequency. When the frequency of electromagnetic wave is low, the induced electromotive force is weak. When the frequency of electromagnetic wave is higher, the attenuation constant of electric field increases and the induced electromotive force is weaker. Therefore, there is an appropriate carrier frequency to maximize the induced electromotive force of the receiving coil, which is the optimal carrier frequency.

Finally, the design of wireless short-range communication system is discussed. The system consists of two parts, one is rotary steering drilling system, and the other is the short-range wireless communication system.

Rotary steering drilling system includes surface monitoring system, measurement drilling system and downhole rotary steering drilling tools, as shown in the Figure below:

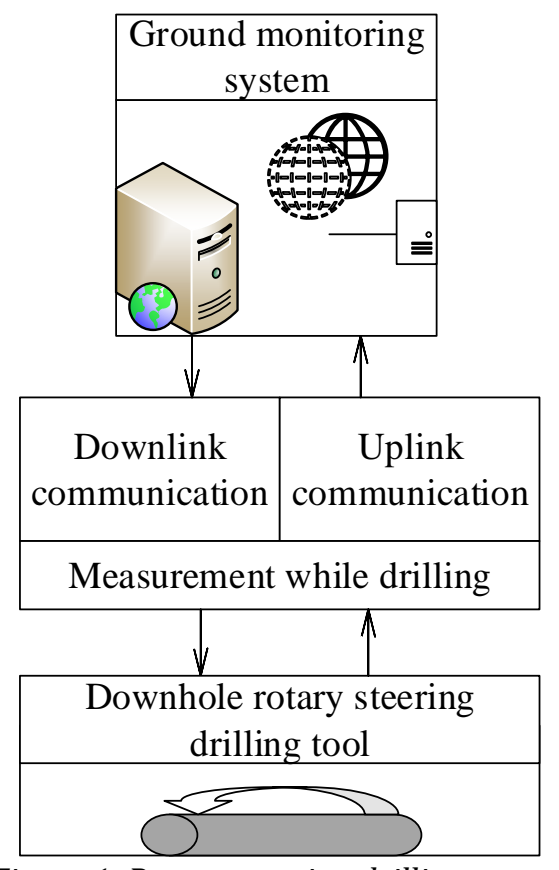

Figure 1: Rotary steering drilling system

A detection circuit is set in the control mechanism of downhole rotary steering drilling tool to detect downhole data. The data, such as well deviation angle, tool face angle and azimuth angle, are detected by sensors and stored in E2PROM of control mechanism [19]. These data need to be transmitted to the ground monitoring system through uplink communication for analysis. Through the analysis of the ground monitoring system, the rotary trajectory of the drill bit is controlled by the downlink communication to realize the strong closed-loop control. The control mechanism rotates relative to the MWD system, which is suitable for transmitting the downhole data to the MWD system through wireless transmission, and then transmitting the MWD to the ground monitoring system through mud pulse fluid.

The structure of near bit wireless proximity communication system is shown in Figure 2.

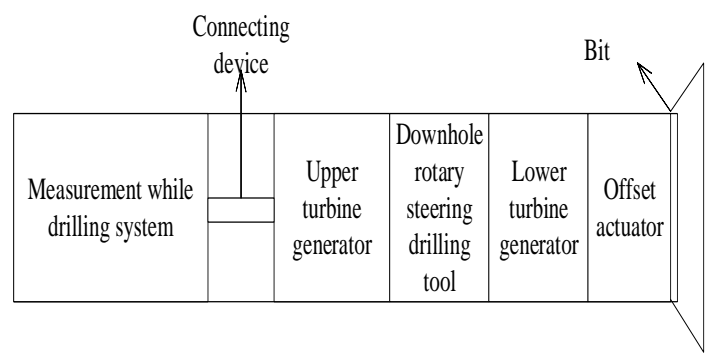

Figure 2: Components of wireless short distance communication system

Wireless short distance communication system mainly includes MWD system and downhole guide drilling tool. The receiving system is installed in the MWD system. The receiving system includes receiving coil and receiving circuit. The receiving circuit demodulates the sinusoidal carrier received by the receiving coil to obtain the near bit information. The downhole steering drilling tool is mainly composed of upper turbine generator, electronic bin and lower turbine generator. The launching system is placed in the electronic bin, including launching circuit, launching coil and detection circuit. The transmitting circuit modulates the data detected by the detecting circuit and transmits it through the radiation coil. This research is to send the near data in the electronic warehouse to the MWD system through short wave wireless transmission.

\subsection{Mechanical Fault Prediction based on Scattering Transform and a Wireless Sensor Network}

First, the principle of a wireless sensor network is displayed. A wireless sensor network system usually includes sensor node, sink node and management node. A wireless sensor network node is usually a micro embedded system, and its data processing capacity, storage capacity and communication capacity are relatively weak. Furthermore, the power supply is provided by the battery carrying limited energy [20]. In a wireless sensor network, sink node has better processing ability, storage ability and communication ability than general node. General node is responsible for connecting a wireless sensor network with external network like Internet, realizing communication protocol conversion between different protocol 
stacks, completing monitoring task of management node, and forwarding collected data to external network. Sink node can be a sensor node with enhanced function, sufficient energy supply and more memory and computing resources, or a special gateway device with wireless communication interface without monitoring function.

Additionally, a wireless sensor network system is designed. At present, there are not a complete system analysis and research results in the application of a wireless sensor network in mechanical equipment fault monitoring and diagnosis. In order to study the application system further, the system structure of a wireless sensor network for mechanical fault diagnosis is proposed. The structure model of the system is shown in Figure 3:

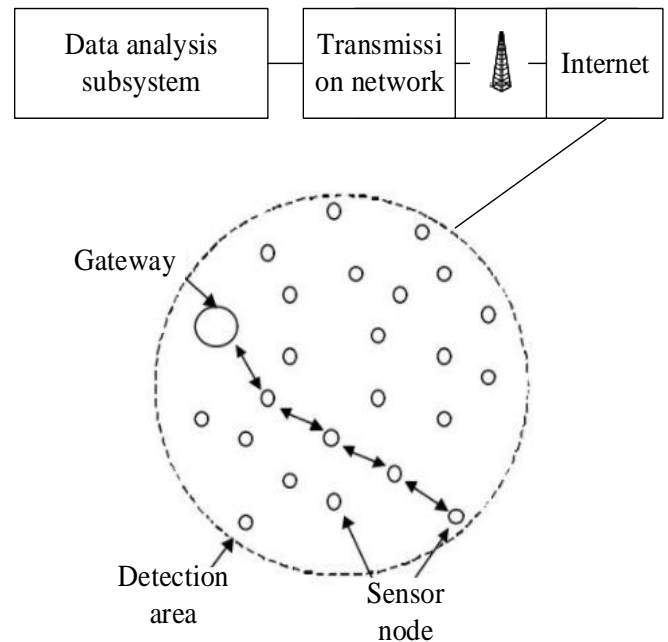

Figure 3: Structure of mechanical fault detection system based on A wireless sensor network

The system is a hierarchical network structure, and the lowest layer is the sensor nodes installed in the monitoring system. The sink node collects the data sent by the sensor nodes and connects to the base station through the transmission network after data fusion. Finally, the base station connects the mechanical equipment fault monitoring and diagnosis results to the remote users through the Internet. A wireless sensor network not only complements the shortcomings of traditional wired system, but also focuses on wireless communication, distributed data acquisition and processing and centralized information processing, which saves installation costs, enhances the fault tolerance of the system and increases flexibility [21, 22]. When the accurate monitoring position cannot be determined, the monitoring effect of the sensor attached to the monitoring position is better than that of using only a single sensor.
Finally, the signal processing based on scattering transform is introduced. It mainly includes two parts, one is fault feature extraction, and the other is fault prediction. The accuracy of feature extraction directly affects the result of fault recognition.

Therefore, effective fault feature extraction is the key to mechanical fault diagnosis. The feature of mechanical fault is obtained by scattering transformation. Because of the translation invariance and deformation stability of the scattering transform, the essential features of mechanical faults can be extracted. Due to the energy conservation of scattering transformation, the energy of each subband of scattering coefficient is taken as the fault feature. The scattering coefficients are set from left to right and from top to bottom and they are $A_{1}, A_{2}, \ldots A_{7}$. The energy formalization of each subband coefficient can be expressed as:

$$
E_{q}=\sum_{x=1}^{\mathrm{r}} A_{q, x}^{2}
$$

$q$ is the number of scattering coefficients and $r$ is the number of all summation coefficients.

The eigenvectors $\left[E_{1}, E_{2}, \ldots E_{7}\right]$ based on each subband are formed. Then, the feature is used for further classification.

In fault diagnosis, the selection of classifier is the key. LSPTSVM is a double classifier [23]. In order to solve the multi classification problem, ensemble learning structure is adopted. Its basic classifier is LSPTSVM, which uses the common one-to-one structure and voting mechanism is to form a multi classifier for fault prediction, as shown in Figure 4 below:

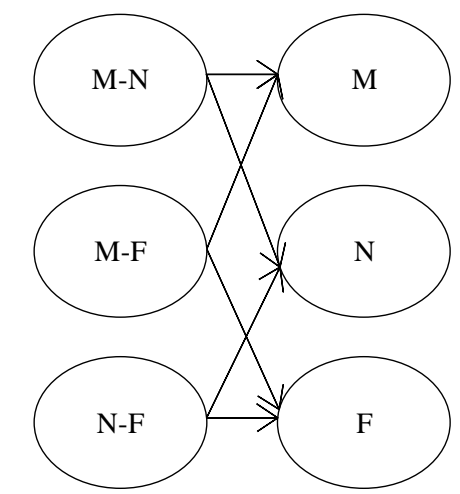

Figure. 4 One to one classification

\subsection{Simulation Experiment}

(1) The relationship between EMF of receiving coil and carrier frequency under different conductivity is analysed by MATLAB simulation. The parameter selection is shown in Table 3: 
Table 3 Communication simulation parameters

\begin{tabular}{cc}
\hline Name of parameters & Value \\
\hline $\mathrm{I}$ & $2 \mathrm{~A}$ \\
$\lambda$ & 1 \\
$\mathrm{~A}$ & $0.02 \mathrm{~m}^{2}$ \\
$\mathrm{M}$ & 150 \\
\hline
\end{tabular}

(2) The hierarchical structure of scattering transform is composed of iterative wavelet mode operation and low-pass filtering [24]. The order of scattering transformation determines the depth of scattering transformation hierarchy and the number of subband. If the value is too small, the amount of information of the average signal after low-pass filtering will be too weak, resulting in low classification accuracy; if it is too strong, the structure of the scattering transform will become very complex. Additionally, the energy contained in the high-order coefficients will be reduced, and the contribution to classification will not be great. In terms of energy conservation, the order of scattering transformation is 2, and the energy ratio is more than $99 \%$ [25].

(3) Origin 2018 64bit is used to analyse the data.

\section{System Performance Simulation and Mechanical Experiment Results}

3.1 Relationship between the Induced Electromotive Force and Carrier Frequency of the Medium

The relationship between the induced electromotive force of the receiving coil and carrier frequency under different conductivity is visualized, and the results are shown in Figure 5 below:

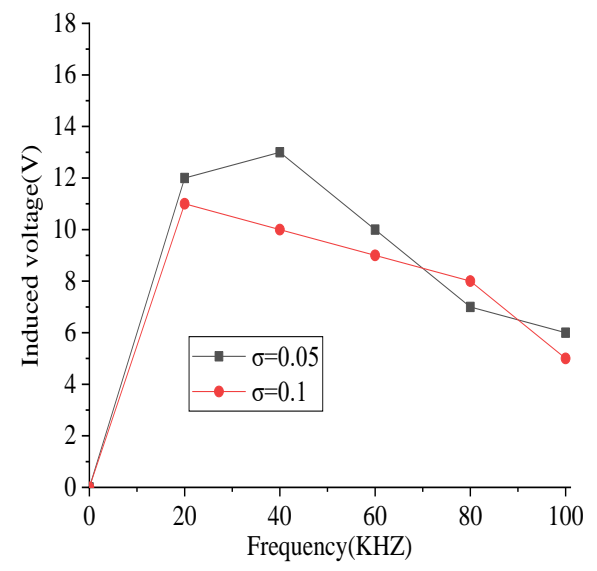

Figure 5: Relationship between induced voltage and frequency under different conductivity

The figure shows that the induced voltage first increases and then decreases with the increase of frequency under different conductivity conditions. When the conductivity is 0.05 , the optimal carrier frequency is $13 \mathrm{KHz}$; and when the conductivity increases to 0.1 , the optimal carrier frequency is $1113 \mathrm{KHz}$, indicating that when the conductivity increases, the optimal carrier frequency will decrease.

\subsection{Fault Prediction Performance of Scatter Transform}

The performance of scattering change and fault prediction is analysed visually, and the results are shown in figure $6 \mathrm{AB}$ below:
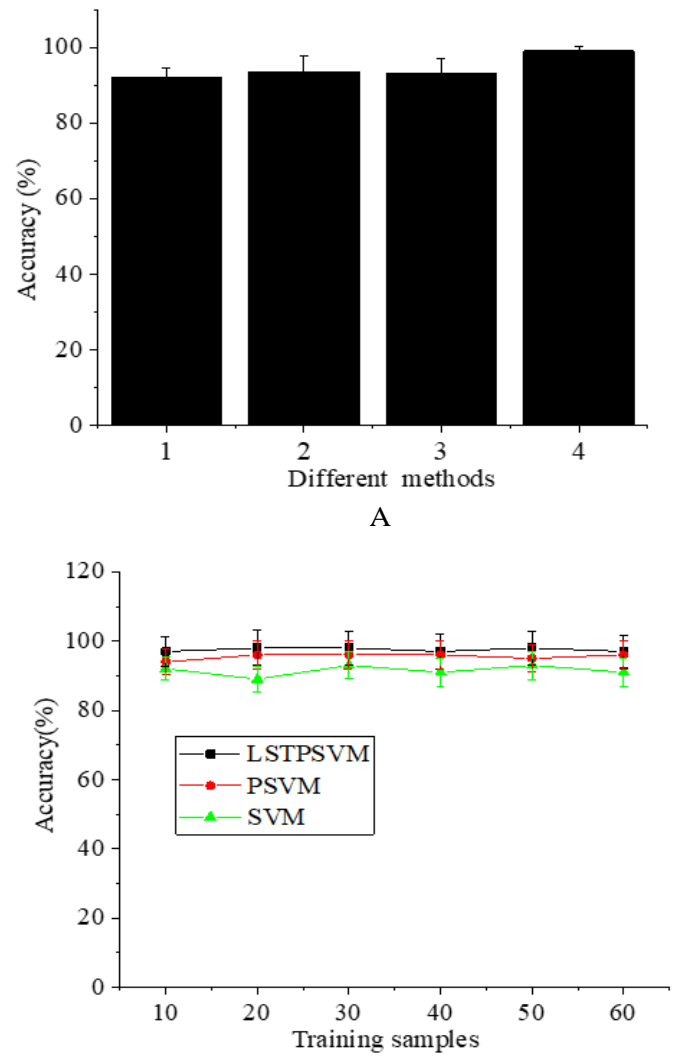

B

Figure 6: Feature representation and fault prediction ability of scattering change (A: feature representation 1: wavelet transform 2: optimized wavelet transform 3: dual tree wavelet transform 4: scattering transform B: fault prediction ability PSVM: standard approximate support vector machine LSTPSVM: least squares projection dual support vector machine SVM: support vector machine)

Figure A indicates that under non-noise situation, the accuracy of the scattering change method used is higher than that of the other three feature extraction methods, exceeding 99\%, while the accuracy of the other three feature extraction methods is about 95\%. Figure B tells that when the number of training samples is more than 20, the quantitative accuracy of STPSVM, PSVM and SVM is 99\%, 96\% and 94\% 
respectively. In different training sets, LSTPSVM has the highest accuracy and smaller variance, which indicates that the classification performance of LSTPSVM is much better than PSVM and SVM in equipment fault prediction.

\subsection{Results of Wireless Transmission Distance with the Change of Electromagnetic Wave Frequency}

The change of wireless transmission distance under the condition of constant change of electromagnetic wave frequency is analysed, and the results are shown in the figure $7 \mathrm{AB}$ below:

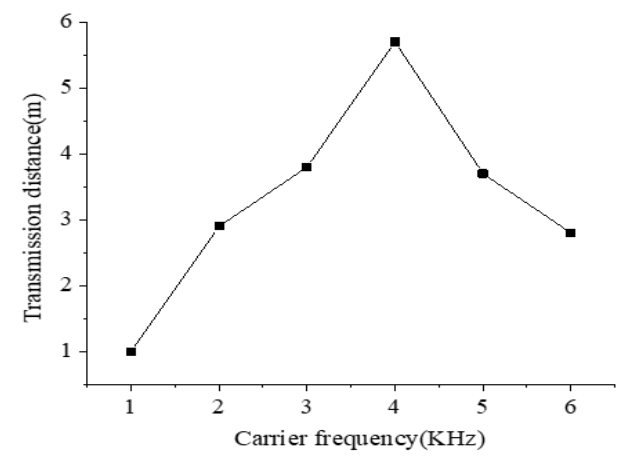

A

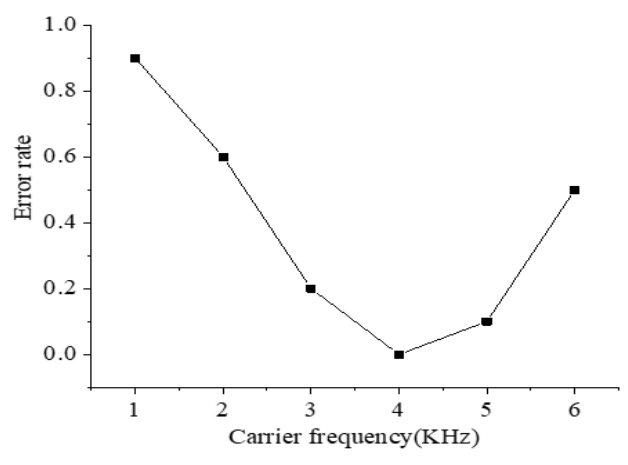

B

Figure 7: Transmission distance and bit error rate under different carrier frequencies (A: transmission distance B: bit error rate 1: (5 10) 2: (10 20) 3: (20 30) 4: (30 40) 5: (40 50) 6: (50 60)

The figure above shows that the distance increases with the increase of carrier frequency. When the carrier frequency is $30 \mathrm{KHZ}$ and $40 \mathrm{KHZ}$ respectively, the maximum transmission distance reaches $5.9 \mathrm{~m}$, and then decreases with the increase of carrier frequency. This indicates the proposed wireless communication system can choose the best carrier frequency. Figure B displays when the distance between the transmitting system and the receiving system is $5.9 \mathrm{~m}$, and the bit error rate begins to decrease with the increase of carrier frequency. When the carrier frequency is $30 \mathrm{KHZ}$ and $40 \mathrm{KHZ}$, the BER is 0 , and then increases with the increase of carrier frequency.

\subsection{Results Analysis of Mechanical Vibration Signals under Different Frequencies}

The normal signal and fault signal of the machine are compared and analysed, and the results are shown in the figure $8 \mathrm{AB}$ below:

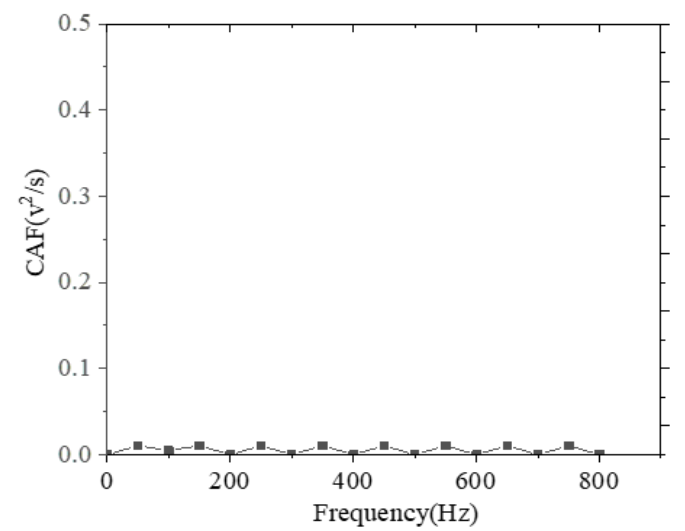

A

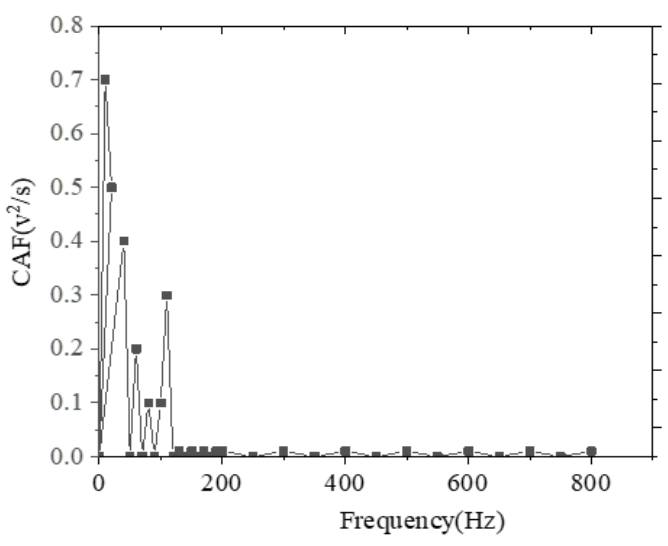

B

Figure 8: Normal signal and fault signal of mechanical operation (A: normal signal, B: fault signal)

Figure $8 \mathrm{~A}$ above tells that the frequency signal of normal operation of the machine is very stable. Figure $8 \mathrm{~B}$ demonstrates that the weak characteristic frequency of $12 \mathrm{~Hz}$ is extracted from the fault power spectrum, which occupies a significant position compared with other frequency components. This shows that the proposed method can effectively suppress other frequency components in the signal, extract the characteristics of periodic fault signal, achieving the purpose of feature extraction.

\section{Conclusion}

Aiming at the technical problems encountered in oil exploitation, the underground electromagnetic wave transmission is discussed based on the wireless short-range communication system and the principle of electromagnetic induction and underground short-range wireless communication. 
In accordance with the principle of scattering transform and a wireless sensor network, the scattering mechanical fault prediction is carried out, and the fault feature extraction and fault prediction are conducted through the signal processing of scattering transform.

The simulation results show that the induced voltage first increases and then decreases with the increase of frequency under different conductivity conditions, and the optimal carrier frequency decreases with the increase of conductivity. Under the condition without noise, the accuracy of the scattering transform method used is more than $99 \%$. In different training sets, LSTPSVM has the highest accuracy and small variance, that is to say, the classification performance of LSTPSVM is much better than SVM and PSVM in equipment fault prediction. The wireless transmission distance first increases with the increase of carrier frequency, and then decreases with the increase of carrier frequency. The change of BER is opposite to the transmission distance.

The proposed wireless communication system can select the best carrier frequency, effectively suppress other frequency components in the signal, and extract the characteristics of periodic fault signal, realizing the purpose of feature extraction. Some shortcomings are summarized as follows: the fusion performance of the sensor equipment involved in the system is relatively weak, and the performance of the system is suitable for the ground simulation. Whether the system can be fully applied to the underground operation needs further verification, which is also the focus for further studies.

\section{References}

[1] Rangel-Alvarado R B, Willis C E, Kirk J L, et al. (2019) Athabasca oil sands region snow contains efficient micron and nano-sized ice nucleating particles. Environmental Pollution, 252, 289-295.

[2] Barbakov O M, Belonozhko M L, Silin A N, et al. (2018) Development of Oil and Gas Resources of Russia's Arctic zone. Journal of the Geological Society of India, 92(6), 764-766.

[3] Jennings M. (2019) The oil and gas industry, the Offshore Installation Manager (OIM) and the management of emergencies - Changes necessary to achieve effective competence assessment of OIMs in controlling emergencies. Journal of Loss Prevention in the Process Industries, 59, 1-13.

[4] Kuo C L, Soo S L, Aspinwall D K, et al. (2018) Development of single step drilling technology for multilayer metallic-composite stacks using uncoated and PVD coated carbide tools. Journal of Manufacturing Processes, 31(JAN.), 286-300.
[5] Shaoran R, Yanmin L, Zhiwu G, et al. (2018) Numerical simulation of water and sand blowouts when penetrating through shallow water flow formations in deep water drilling. Journal of Ocean University of China, 17(001), 1724.

[6] Oparin V N, Timonin V V, Karpov V N, et al. (2017) Energy-Based Volumetric Rock Destruction Criterion in the Rotary-Percussion Drilling Technology Improvement. Journal of Mining Science, 53(6), 1043-1064.

[7] Tian W, Zhou Z, Liao W. (2016) Analysis and investigation of a rivet feeding tube in an aircraft automatic drilling and riveting system. The International Journal of Advanced Manufacturing Technology, 82(5-8), 973-983.

[8] Shehata M, Mostafa H, Ismail Y. (2017) Accurate Closed Form Expressions for The Bit RateWireless Transmission Distance Relationship in IR-UWBoF Systems. IEEE Communications Letters, PP(10), 1-1.

[9] Kikuchi N. (2019) Multilevel Signaling Technology for Increasing Transmission Capacity in High-Speed Short-Distance Optical Fiber Communication. IEICE Transactions on Electronics, E102.C(4), 316-323.

[10] Bakhtiari-Nejad F, Hosseinzadeh A. (2017) Nonlinear dynamic stability analysis of the coupled axial-torsional motion of the rotary drilling considering the effect of axial rigid-body dynamics. International Journal of Non-Linear Mechanics, 88(jan.), 85-96.

[11] Krainyukov A, Krivchenkov A, Saltanovs R. (2017) Performance Analysis of Wireless Communications for V2G Applications Using WPT Technology in Energy Transfer. Procedia Engineering, 178(Complete), 172-181.

[12] Abayaje F, Febvre P. (2016) A Customized Reduced Size Antipodal Vivaldi Antenna used in Wireless Baseband Transmission for Short-Range Communication. AEU - International Journal of Electronics and Communications, 70(12), 16841691.

[13] Lee B C, Chung G S. (2017) Design and analysis of a pendulum-based electromagnetic energy harvester using anti-phase motion. Iet Renewable Power Generation, 10(10), 1625-1630.

[14] Najafi A, Iskender I. (2016) A new approach to reduce the leakage flux and electromagnetic force on distribution transformer under unbalanced faults based on finite element method. International Transactions on Electrical Energy Systems, 26(4), 901-916.

[15] Alarcón, J M, Weiss C. (2018) Accurate nucleon electromagnetic form factors from dispersively improved chiral effective field theory. Physics Letters B, 784, 373-377. 
[16] Underwood M K, Ehrenreich S E. (2017) The power and the pain of adolescents' digital communication: Cyber victimization and the perils of lurking. Am Psychol, 72(2), 144-158.

[17] Grote M J, Schneebeli A, Schotzau D. (2018) Interior penalty discontinuous Galerkin method for Maxwell's equations: optimal L2-norm error estimates. Ima Journal of Numerical Analysis, 204( 2), 375-386.

[18] Najari S, Jazi B. (2020) The description of mode matching method, in electromagnetic wave transmission from a loss free semi-bounded waveguide to the plasma waveguide. The European Physical Journal Plus, 135(10), 1-10.

[19] Weaver J W, Murray A R, Kremer F V. (2017) Estimation of the proximity of private domestic wells to underground storage tanks: Oklahoma pilot study. The Science of the Total Environment, 609(dec.31), 1589-1599.

[20] Zhang, Q. (2016) Cumulated Checksum and Dynamic Addresses for Secure A wireless sensor networks. International Journal of Distributed Sensor Networks, 12(7), 3984961-3984961.
[21] Ferng H W, Khoa N M. (2017) On security of a wireless sensor networks: a data authentication protocol using digital signature. Wireless Networks, 23(4), 1113-1131.

[22] Ivanov S, Balasubramaniam S, Botvich D, et al. (2016) Gravity gradient routing for information delivery in fog A wireless sensor networks. Ad Hoc Networks, 46(AUG.), 61-74.

[23] Tanveer M, Khan M A, Ho S S. (2016) Robust energy-based least squares twin support vector machines. Applied Intelligence, 45(1), 174-186.

[24] Martin T P, Rohde C A, Guild M D, et al. (2016) Acoustic Scattering Cancellation: an Alternative to Coordinate Transformation Scattering Reduction. Journal of the Acoustical Society of America, 139(4), 2183-2183.

[25] Ma X, Wang J. (2019) Transformations of basic hypergeometric series from the viewpoint of substitution of parameter operators. The Ramanujan Journal, 50(1), 61-91. 